\title{
ON FOURIER TRANSFORMS
}

BY

\section{NASIM}

ABSTRACT. If $f(x)$ and $g(x)$ satisfy the equations

$$
g(x)=\frac{d}{d x} \int_{0}^{\infty} \frac{1}{t} f(t) k_{1}(x t) d t, \quad f(x)=\frac{d}{d x} \int_{0}^{\infty} \frac{1}{t} g(t) k_{1}(x t) d t,
$$

then we call $f$ and $g$ a pair of $k_{1}$-tran sforms, where

$$
k_{1}=\frac{1}{2 \pi i} \int_{1 / 2-i \infty}^{1 / 2+i \infty} \frac{K(s)}{1-s} x^{1-s} d s .
$$

In this paper altemative sets of conditions are established for $f$ and $g$ to be $k_{1}$-transform provided $K(s)$ is decomposable in a special way. These conditions involve simpler functions, which replace the kernel $k_{1}(x)$. Results are proved for the function spaces $L^{2}$. The necessary and sufficient conditions are established for the two functions to be self-reciprocal. Conditions are given for generating pairs of transforms for a given kernel. Two examples are given at the end to illustrate the methods and the advantage of the results.

1. Introduction. Iterations of the Laplace transforms [9] are well known and are of the form,

$$
g(x)=\int_{0}^{\infty} e^{-x t} f(t) d t, \quad f(x)=\int_{0}^{\infty} e^{-x t} b(t) d t, \quad g(x)=\int_{0}^{\infty} \frac{b(t)}{x+t} d t .
$$

Charles Fox [3] calls this system a chain transform of order 3. He generalizes this to the chain transform of order $n$ by the equations

$$
g_{p+1}(x)=\int_{0}^{\infty} \frac{1}{t} r_{p}(x / t) g_{p}(t) d t, \quad g_{q}(x)=\int_{0}^{\infty} \frac{1}{t} l_{q}(x / t) g_{q+1}(t) d t
$$

and

$$
g_{n+1}(x)=g_{1}(x)
$$

where $p$ and $q$ run through first $n$ integers. He points out that in order to prove this, all the kernels $l_{q}$ and $r_{p}$ must be known along with one of $g_{i}(x)$ functions, cf. [7].

We shall consider the iteration of Laplace transforms from a different point of "view. Let us consider the following formal result. Let

Received by the editors J anuary 17, 1973 and, in revised form, May 18, 1973. AMS (MOS) subject classifications (1970). Primary 42A68, 44A05; Secondary 44A35.

Key words and phrases. Fourier transform, Fourier kernel, Mellin transform, $L^{2}$-class, convergence in mean, the Parseval theorem, Bessel functions.

Copyrieht $\odot 1974$, American Mathematical Society 


$$
g(x)=\int_{0}^{\infty} \phi(t) l(x t) d t \text { and } f(x)=\int_{0}^{\infty} \frac{1}{t} \phi\left(\frac{1}{t}\right) m(x t) d t .
$$

If $K(s)=L(s) / M(1-s)$, then $g(x)=\int_{0}^{\infty} f(t) k(x t) d t$. Here $K(s), L(s)$ and $M(s)$ denote the Mellin transforms of $k(x), l(x)$ and $m(x)$ respectively. The system of equations (1), becomes a special case of this if we put

$$
\frac{1}{x} \phi\left(\frac{1}{x}\right)=b(x), \quad m(x)=e^{-x}, \quad l(x)=\frac{1}{1+x} \text { and } k(x)=e^{-x} \text {. }
$$

If we assume further that $L(s) L(1-s)=M(s) M(1-s)$, then it follows that $K(s) K(1-s)=1$ and $k(x)$ is then a Fourier kernel. Thus the system of equations (2), implies that

$$
g(x)=\int_{0}^{\infty} f(t) k(x t) d t \text { and } f(x)=\int_{0}^{\infty} g(t) k(x t) d t .
$$

In other words for a pair of functions $f(x)$ and $g(x)$ to be Fourier transforms of each other with respect to the kernel $k(x)$, it is necessary that they satisfy the equations (2) for some $\phi(x)$. Thus we have an alternative way of expressing the relationship between $f(x)$ and $g(x)$. For example, let

$$
g(x)=\int_{0}^{\infty} e^{-1 / 2 x^{2} t^{2}} \phi(t) d t \text { and } f(x)=\int_{0}^{\infty} e^{-1 / 2 x^{2} t^{2} \cdot \frac{1}{t} \phi}\left(\frac{1}{t}\right) d t
$$

for some $\phi(x)$. Then [4],

$$
g(x)=2 \pi \int_{0}^{\infty} f(t) \cos x t d t \text { and } f(x)=2 \pi \int_{0}^{\infty} g(t) \cos x t d t,
$$

since the Mellin transform of $\cos x$ is given by $L(s) / L(1-s)$ where $L(s)$ is the Mellin transform of $e^{-x^{2} / 2}$. The advantage of this set of conditions for a pair of functions, is that the integrals involve simpler and elementary functions, thus replacing a somewhat unwieldy function $k(x)$. In most cases the integrals in these new conditions can simply be read from the tables. Also, numerous sets of pairs of transforms for a given kernel can be generated by varying $\phi(x)$ in equation (2). For instance putting $\phi(x)=x e^{-x^{2 / 2}}$ in (3), we get a well-known pair of cosine transforms $e^{-x}, 1 /\left(1+x^{2}\right)[1$, p. 146, (28)].

In this paper we shall establish results dealing with these new conditions for a pair of Fourier transforms, for the function class $L^{2}(0, \infty)$.

2. Preliminaries. (i) Notations. We shall use the following notations:

(1) $(1 / 2 \pi i) \int_{1 / 2}$ for l.i.m. $T \rightarrow \infty \int_{1 / 2-i T}^{1 / 2+i T}$, where l.i.m. stands for limit in the mean.

(2) $L^{p}(0, \infty)$ will be the Lebesgue integrable class, $p \geq 1$.

(3) $J_{v}, K_{v}, Y_{v}$ are the usual Bessel functions of order $v$.

(ii) Known results. (A) Let $f(x) \in L^{2}(0, \infty)$, then $F(s)=\int_{0}^{\infty} f(x) x^{s-1} d x$ . exists in the mean square sense and $F(s) \in L^{2}(1 / 2-i \infty, 1 / 2+i \infty)$. If $F(s) \epsilon$ $L^{2}(1 / 2-i \infty, 1 / 2+i \infty)$, then $f(x)=(1 / 2 \pi i) \int_{1 / 2} F(s) x^{-s} d s$, and $f(x) \in L^{2}(0, \infty)$. We call $F(s)$ the Mellin transform of $f(x)$. 
(B) The Parseval theorem. Let $f(x)$ and $g(x) \in L^{2}(0, \infty)$; then

$$
\int_{0}^{\infty} f(x) g(x) d x=\frac{1}{2 \pi i} \int_{1 / 2-i \infty}^{1 / 2+i \infty} F(s) G(1-s) d s,
$$

where $F(s)$ and $G(s)$ are the Mellin transforms of $f(x)$ and $g(x)$, respectively.

(C) Let $K(s)$ be any function satisfying $|K(s)|=1, K(s) K(1-s)=1$ on $s=$ $1 / 2+i t$, all real $t$. Let $k_{1}(x)=(1 / 2 \pi i) \int_{1 / 2} K(s) x^{1-s} /(1-s) d s$, and $f(x) \in L^{2}(0, \infty)$, then

$$
g(x)=\frac{d}{d x} \int_{0}^{\infty} f(t) k_{1}(x t) \frac{d t}{t}, \text { a.e., } x>0
$$

and $g(x) \in L^{2}(0, \infty)$. Further

$$
f(x)=\frac{d}{d x} \int_{0}^{\infty} g(t) k_{1}(x t) \frac{d t}{t} \text { a.e. }
$$

We shall call $f(x)$ and $g(x) k_{1}$-transforms of each other. These are known results, and can be obtained as special cases or simple modifications of results in Titchmarsh [8].

3. Properties in the class $L^{2}$. We shall now prove two theorems for transforms of $L^{2}$-class.

Theorem 1. Let $L(s)$ and $M(s)$ be such that (i) $L(s) \neq 0$ and is bounded on the line $s=1 / 2+i t,-\infty<t<\infty$, and (ii) $L(s) L(1-s)=M(s) M(1-s)$. Let there exist a function $\phi(x) \in L^{2}(0, \infty)$. Define functions $f(x)$ and $g(x)$ by the equations (iii) $G(s)=L(s) \Phi(1-s)$, (iv) $F(s)=M(s), \Phi(s)$, where $\Phi(s), F(s)$ and $G(s)$ are the Mellin transforms of $\phi(x), f(x)$ and $g(x)$ respectively. Then $f(x)$ and $g(x) \epsilon$ $L^{2}(0, \infty)$ and

$$
\int_{0}^{x} g(t) d t=\int_{0}^{\infty} \frac{1}{t} l_{1}(x t) \phi(t) d t, \quad \int_{0}^{x} f(t) d t=\int_{0}^{\infty} \frac{1}{t^{2}} m_{1}(x t) \phi\left(t^{-1}\right) d t
$$

for all $x>0$, where

$$
l_{1}(x)=\frac{1}{2 \pi i} \int_{1 / 2} \frac{L(s)}{1-s} x^{1-s} d s, \quad m_{1}(x)=\frac{1}{2 \pi i} \int_{1 / 2} \frac{M(s)}{1-s} x^{1-s} d s .
$$

Further if $(v) K(s)=L(s) / M(1-s)$, then $f(x)$ and $g(x)$ are $k_{1}$-transforms of each other as in the result (C).

Proof. Since $\phi(x) \epsilon L^{2}(0, \infty)$, therefore its Mellin transform $\Phi(s) \epsilon$ $L^{2}(1 / 2-i \infty, 1 / 2+i \infty)$, due to the result (A). Now from the conditions (i) and (iii), we deduce that $G(s) \in L^{2}(1 / 2-i \infty, 1 / 2+i \infty)$, hence $g(x)$, whose Mellin transform is $G(s)$ must belong to $L^{2}(0, \infty)$, as required. Note that from (i) and (ii), we conclude that $M(s) \neq 0$ and is bounded on the line $s=1 / 2+i t$, and therefore using (iv), as before, we can show that $F(s) \in L^{2}(1 / 2-i \infty, 1 / 2+i \infty)$ and consequently $f(x) \in L^{2}(0, \infty)$. The Mellin transform of $b(t)=1(t<x)$ and $b(t)=0(t>x)$ is $H(s)=x^{s} / s$. On multiplying the equation in (iii) by $H(1-s)$ and integrating we obtain 


$$
\frac{1}{2 \pi i} \int_{1 / 2} G(s) \frac{x^{1-s}}{1-s} d s=\frac{1}{2 \pi i} \int_{1 / 2} L(s) \Phi(1-s) \frac{x^{1-s}}{1-s} d s .
$$

Now from (i), we obtain that $\left.(L(s) /(1-s))=\alpha_{t^{-1}}\right)$, therefore $L(s) /(1-s) \epsilon$ $L^{2}(1 / 2-i \infty, 1 / 2+i \infty)$ and so from (5), $l_{1}(x) / x$, whose Mellin transform is $L(s) /(1-s)$, also $\in L^{2}(0, \infty)$. Thus using the result (B), we have from above,

$$
\int_{0}^{x} g(t) d t=\int_{0}^{\infty} \frac{1}{t} l_{1}(x t) \phi(t) d t
$$

as required. Similarly starting from the equation in (iv) and multiplying it by $H(1-s)$, and using the result (B), we obtain

$$
\int_{0}^{x} f(t) d t=\int_{0}^{\infty} \frac{1}{t^{2}} m_{1}(x t) \phi\left(t^{-1}\right) d t
$$

Now replace $s$ by $1-s$ in (iv) and divide into (iii) to eliminate $\Phi(1-s)$, to get

$$
G(s)=F(1-s) L(s) / M(1-s)=F(1-s) K(s),
$$

using (v). From (6), we obtain the following equation

$$
\frac{1}{2 \pi i} \int_{1 / 2} G(s) \frac{x^{1-s}}{1-s} d s=\frac{1}{2 \pi i} \int_{1 / 2} F(1-s) K(s) \frac{x^{1-s}}{1-s} d s .
$$

By using (i) and (v), we have $|K(s) /(1-s)|=O\left(t^{-1}\right)$, therefore $K(s) /(1-s) \epsilon$ $L^{2}(1 / 2-i \infty, 1 / 2+i \infty)$ and consequently $k_{1}(x) / x \in L^{2}(0, \infty)$. And by the result (B), equation (7) yields

$$
\int_{0}^{x} g(t) d t=\int_{0}^{\infty} \frac{1}{t} f(t) k_{1}(x t) d t .
$$

Next, replace $s$ by $1-s$ in (iii) and divide into (iv) to obtain

$$
F(s)=G(1-s) M(s) / L(1-s)=G(1-s) K(s) .
$$

As before, this equation gives rise to the equation

$$
\frac{1}{2 \pi i} \int_{1 / 2} F(s) \frac{x^{1-s}}{1-s} d s=\frac{1}{2 \pi i} \int_{1 / 2} G(1-s) K(s) \frac{x^{1-s}}{1-s} d s,
$$

which yields, using the result $(B)$, the equation

$$
\int_{0}^{x} f(t) d t=\int_{0}^{\infty} \frac{1}{t} g(t) k_{1}(x t) d t .
$$

Hence from (8) and (9) we conclude that $f(x)$ and $g(x)$ are $k_{1}$-transforms of each other.

Next we shall consider some special cases of Theorem 1.

A most general solution of the equations $L(s) L(1-s)=M(s) M(1-s)$ and $|L(1 / 2+i t)|=|M(1 / 2-i t)|$ is $M(s)=d(s) b(s)$ and $L(s)=d(s) b(1-s)$.

The two extreme cases are when $d(s) \equiv 1$ and $b(s) \equiv 1$.

Case 1. Let $d(s) \equiv 1$. Then $L(s)=M(1-s)$ and $K(s)=1$, whence $k_{1}(x)=$ $(2 \pi i)^{-1} \int_{1 / 2} x^{1-s} /(1-s) d s$. Now (9) gives 


$$
\begin{aligned}
\int_{0}^{x} f(t) d t & =\int_{0}^{\infty} \frac{1}{t} k_{1}(x t) g(t) d t \\
& =\frac{1}{2 \pi i} \int_{1 / 2-i \infty}^{1 / 2+i \infty} \frac{G(1-s)}{1-s} x^{1-s} d s=\int_{0}^{x} \frac{1}{t} g\left(t^{-1}\right) d t
\end{aligned}
$$

by Parseval's theorem for Mellin transforms. Hence $f(x)=x^{-1} g\left(x^{-1}\right)$ a.e. Similarly (8) gives $g(x)=x^{-1} f\left(x^{-1}\right)$ a.e., which are clearly consequences of one another $[8$, p. 218].

Case 2. Let $b(s) \equiv 1$. Then $L(s)=M(s)$ and $K(s)=L(s) / L(1-s)$. Thus

Corollary 1. Let $l_{1}(x)$ and $\phi(x)$ satisfy the conditions of Theorem 1. Define $f(x)$ and $g(x)$, both $\in L^{2}(0, \infty)$, by (4). Then $f(x)$ and $g(x)$ are a pair of $k_{1}$-trans. forms, with

$$
k_{1}(x)=\frac{1}{2 \pi i} \int_{1 / 2} \frac{K(s)}{1-s} x^{1-s} d s \text { and } K(s)=\frac{L(s)}{L(1-s)} .
$$

If we choose $\phi(x)$ to be such that $\phi(x)=x^{-1} \phi\left(x^{-1}\right)$, the equations (4) give us a self-reciprocal function with respect to the kernel $k_{1}(x)$ [8].

Next we shall prove a converse of Theorem 1.

Theorem 2. Let $f(x)$ and $g(x)$ be $k_{1}$-transforms of each other according to the result (C). Let (i) $K(s)=M(s) / L(1-s)$ where (ii) $L(1-s)$ is bounded on the line $s=1 / 2+i t,-\infty<t<\infty$. If (iii) $\Phi(s)=G(1-s) / L(1-s)$, then

$$
\int_{0}^{x} f(t) d t=\int_{0}^{\infty} \frac{1}{t^{2}} m_{1}(x t) \phi\left(t^{-1}\right) d t, \quad \int_{0}^{x} g(t) d t=\int_{0}^{\infty} \frac{1}{t} l_{1}(x t) \phi(t) d t,
$$

where $\phi(x)$ and $g(x)$ are the functions whose Mellin transforms are $\Phi(s)$ and $G(s)$, and $l_{1}(x), m_{1}(x)$ are defined by (5) above.

Proof. Since $f(x)$ and $g(x)$ are $k_{1}$-transforms of each other therefore, $\int_{0}^{x} f(t) d t=\int_{0}^{\infty} t^{-1} k_{1}(x t) g(t) d t$. Define a function $b(t)=1(t<x)$ and $b(t)=0$ $(t>x)$; its Mellin transform $H(s)=x s / x$. Now from above $\int_{0}^{\infty} f(t) b(t) d t=$ $\int_{0}^{\infty} t^{-1} k_{1}(x t) g(t) d t$. We have seen earlier that $k_{1}(x) / x \in L^{2}(0, \infty)$. Also $b(x) \epsilon$ $L^{2}(0, \infty)$. Therefore by the result (B), the last equation yields,

$$
\frac{1}{2 \pi i} \int_{1 / 2} F(s) \frac{x^{1-s}}{1-s} d s=\frac{1}{2 \pi i} \int_{1 / 2} K(s) G(1-s) \frac{x^{1-s}}{1-s} d s,
$$

where, from the rusult (C), $K(s) /(1-s)$ is the Mellin transform of $k_{1}(t) / t$ and $F(s)$ denotes the Mellin transform of $f(x)$. Thus

$$
F(s)=\frac{M(s) G(1-s)}{L(1-s)}=\Phi(s) M(s) \text {, a.e. }
$$

and therefore $F(s) H(1-s)=\Phi(s) M(s) H(1-s)$ which yields

$$
\frac{1}{2 \pi i} \int_{1 / 2} F(s) \frac{x^{1-s}}{1-s} d s=\frac{1}{2 \pi i} \int_{1 / 2} \Phi(s) M(s) \frac{x^{1-s}}{1-s} d s .
$$


Now $K(s)=M(s) / L(1-s)$ and $K(s) K(1-s)=1$, therefore $L(s) L(1-s)=M(s) M(1-s)$. From (ii) we conclude that $M(s)$ is bounded on $s=1 / 2+i t,-\infty<t<\infty$ and so $|M(s) /(1-s)|=O\left(t^{-1}\right)$. Thus $M(s) /(1-s) \in L^{2(1 / 2-i \infty, 1 / 2+i \infty)}$ which implies that $m_{1}(x) / x$ whose Mellin transform is $M(s) /(1-s)$ by $(5), \epsilon L^{2}(0, \infty)$. Also from (iii) we conclude that $\Phi(s) \in L^{2}(1 / 2-i \infty, 1 / 2+i \infty)$, therefore $\phi(x)$, whose Mellin transform is $\Phi(s), \epsilon L^{2}(0, \infty)$. It is an easy matter to deduce that $\Phi(1-s)$ is the Mellin transform of $t^{-1} \phi\left(t^{-1}\right)$. By the result (B), equation (10) gives

$$
\int_{0}^{\infty} f(t) b(t) d t=\int_{0}^{\infty} t^{-1} \phi\left(t^{-1}\right) t^{-1} m_{1}(x t) d t,
$$

or

$$
\int_{0}^{x} f(t) d t=\int_{0}^{\infty} t^{-2} \phi\left(t^{-1}\right) m_{1}(x t) d t
$$

as required.

Next from (iii) we deduce that $G(s)=\Phi(1-s) L(s)$. By multiplying the above equation by $H(1-s)$, integrating along the line $1 / 2+i t,-\infty<t<\infty$, and applying the result (B), we conclude, as before, that $\int_{0}^{x} g(t) d t=\int_{0}^{\infty} t^{-1} \phi(t) l_{1}(x t) d t$, and hence the theorem.

One can obtain results analogous to those proved in Theorems 1 and 2, but in the nonintegrated form, under suitable conditions. We shall see below a formal derivation of one such result, from Theorem 1.

Suppose we let $l_{1}(x)=\int_{0}^{x}(t) d t$ and $m_{1}(x)=\int_{0}^{x} m(t) d t$. By differentiating the equations (4) and (5), of Theorem 1, we obtain formally that

$$
g(x)=\int_{0}^{\infty} \phi(t) l(x t) d t, \quad f(x)=\int_{0}^{\infty} t^{-1} \phi\left(t^{-1}\right) m(x t) d t,
$$

where $l(x)$ and $m(x)$ would be in some sense of the form

$$
l(x)=\frac{1}{2 \pi i} \int_{1 / 2} L(s) x^{-s} d s \text { and } m(x)=\frac{1}{2 \pi i} \int_{1 / 2} M(s) x^{-s} d s .
$$

Further if $K(s)=L(s) / M(1-s)$, then by differentiating equations (8) and (9), we obtain formally, that

$$
g(x)=\int_{0}^{\infty} f(t) k(x t) d t \text { and } f(x)=\int_{0}^{\infty} g(t) k(x t) d t,
$$

where the kernel $k(x)$ is given by $k(x)=(2 \pi i)^{-1} \int_{1 / 2} K(s) x^{-s} d s$. That is, $f(x)$ and $g(x)$ are $k$-transforms of each other.

5. Examples. (i) If $k(x)=\pi J_{0}\left(2 \pi x^{1 / 2}\right)$, then $l(x)=2 \cos 2 \pi x, m(x)=\sin 1 / 2 \pi x$.

(ii) If $k(x)=x^{1 / 2} J_{v}(x), v \geq-1 / 2$, then $l(x)=x^{v+1 / 2} e^{1 / 2 x^{2}}=m(x)$.

Acknowledgement. Thanks are due to the referee for suggesting changes in the earlier drafts of this paper. This research was supported by a grant from the National Research Council of Canada. 


\section{BIBLIOGRAPHY}

1. A. Erdélyi et al., Tables of integral transforms. Vol. 1, McGraw-Hill, New York, 1954. MR 15, 868.

2. - Tables of integral transforms. Vol. 2, McGraw-Hill, New York, 1954. MR 16, 468.

3. C. Fox, Chain transforms, Proc. Amer. Math. Soc. 5 (1954), 677-688. MR 16, 127.

4. A. P. Guinand, Reciprocal convergence classes for Fourier series and integrals,

Canad. J. Math. 13 (1961), 19-36. MR 23 \# A472.

5. C. Nasim, On the summation formula of Voronoi, Trans. Amer. Math. Soc. 163 (1972), 35-45.

6. T. L. Pearson, Note on the Hardy-Landau summation formula, Canad. Math. Bull. 8 (1965), 717-720. MR 33 \#2616.

7. O. P. Sharma, The H-functions as kernels in chain transforms, Proc. Nat. Inst. Sci. India Part A 34 (1968), 320-325. MR 40 \#3206.

8. E. C. Titchmarsh, Introduction to the theory of Fourier Integrals, Clarendon Press, Oxford, 1937.

9. D. V. Widder, The Laplace transform, Princeton Math. Series, vol. 6, Princeton Univ. Press, Princeton, N. J., 1941. MR 3, 232.

DEPARTMENT OF MATHEMATICS, THE UNIVERSITY OF CALGARY, CALGARY, ALBERTA, CANADA 